\title{
Topical Rifamycin Prophylaxis in Gynecological and Obstetric Surgery
}

\author{
Nahit ATA ${ }^{1}$, Mehmet KULHAN²${ }^{2}$, Nur Gozde KULHAN ${ }^{3}$, Can TURKLER ${ }^{1}$, Ahmet BILGI ${ }^{2}$, Cetin CELIK ${ }^{2}$ \\ Erzincan, Turkey
}

\section{ABSTRACT}

OBJECTIVE: Antibiotic prophylaxis is one of the most important steps to reduce surgical site infections. First-generation cephalosporin (cefazolin) is used prophylactically in the majority of operations. Rifamycin is a broad-spectrum semisynthetic antibiotic that is bactericidal against gram (+) and gram (-) microorganisms. To the best of our knowledge, there are no studies on the use of rifamycin in antibiotic prophylaxis. In this study, we aimed to analyze whether there is a difference between the use of only cefazolin and only rifamycin in terms of surgical site infections.

STUDY DESIGN: One hundred patients were included in this case-control study during the last quarter period of 2017. These patients $(n=100)$ were divided into two groups according to their antibiotic use; 50 patients who received only $1 \mathrm{~g}$ cefazolin constituted Group 1, 50 patients who received only $250 \mathrm{mg}$ topical rifamycin over the incision line based on surgeon's preference constituted Group 2.

RESULTS: The use of prophylactic topical rifamycin reduced the incidence of wound infection. compared with cefazolin. Surgical site infection was detected in $5(10 \%)$ of the patients who received cefazolin, whereas surgical site infection was not observed in patients who received rifamycin $(p=0.022)$.

CONCLUSIONS: The use of topical rifamycin is effective but does not imply that systemic antibiotics should replace prophylaxis. The use of rifamycin would aid in systemic antibiotic prophylaxis.

Keywords: Antibiotic prophylaxis, Cefazolin, Surgical site infection, Topical rifamycin

Gynecol Obstet Reprod Med 2022;28(1):76-81

\section{Introduction}

Surgical site infections (SSI) continue to be a very important and serious problem of modern surgery despite asepsis and antisepsis applications, sterilization methods, developments in operating room conditions, and prophylactic antibiotics. Infections observed at the incision site and in the organs or areas where surgical intervention was performed within the

${ }^{I}$ Erzincan University Medical Faculty Gynaecology and Obstetrics Department Erzincan, Turkey

2 Selcuk University Medical Faculty Gynaecology and Obstetrics Department Konya, Turkey

${ }^{3}$ Konya Education Research Hospital Gynaecology and Obstetrics Department Konya, Turkey

Address of Correspondence: Mehmet Kulhan

Selcuk University Medical Faculty, Gynaecology and Obstetrics Department, 42250 Konya, Turkey

mehmet_kulhan@yahoo.com

Submitted for Publication: 02.03.2021 Revised for Publication: 02.03.2021 Accepted for Publication: 14.08.2021 Online Published: 31.08.2021

ORCID IDs of the authors: NA: 0000-0002-1161-0926

MK: 0000-0002-5478-7510 NGK: 0000-0002-7463-9101

CT: 0000-0003-2716-0322 AB: 0000-0001-8682-1739 CC: 0000-0002-9881-7814

\begin{tabular}{cc}
\hline Quick Response Code: & Access this article online \\
\cline { 2 - 2 } & Website: www.gorm.com.tr \\
& e- mail: info@gorm.com.tr \\
\hline & DOI:10.21613/GORM.2021.1193 \\
\hline
\end{tabular}

How to cite this article: Ata N. Kulhan M. Kulhan NG. Turkler C. Bilgi A. Celik C. Topical Rifamycin Prophylaxis in Gynecological and Obstetric Surgery. Gynecol Obstet Reprod Med. 2022;28(1):76-81 first 30 days following the operation are called SSI (1). Standard definitions were introduced by the Centers for Disease Control and Prevention (CDC) in 1992 and 1998 to diagnose SSI according to certain criteria and to reach more accurate statistical data. According to the standard definitions introduced by this center, SSIs are divided into two groups as incisional and organ/area infections. Incisional wound infections are also classified as superficial and deep incisional wound infections $(2,3)$.

Sources of pathogens responsible for SSIs are often endogenous flora originating from the patient's skin, mucous membranes, or intestinal tract (4). Exogenous flora primarily includes aerobes. Especially gram-positive microorganisms such as Staphylococcus and Streptococcus are observed (1). Staphylococcus aureus is the most common microorganism observed in clean wounds and is often transmitted from the patient's skin flora. Staphylococcus aureus can also be transmitted exogenously from the environment. Polymicrobial anaerobic and aerobic flora is observed in clean-contaminated, contaminated, and dirty wounds (5). Rifamycin has a highly bactericidal effect on the pathogens mentioned above (6). Due to these properties, rifamycin has been used alone for prophylaxis depending on the surgeon's preference in this study.

Antibiotic prophylaxis is one of the most important steps to reduce SSIs (7). First-generation cephalosporin (cefazolin) is used prophylactically in the majority of operations. 
Rifamycin is a broad-spectrum semisynthetic antibiotic that is bactericidal against gram (+) and gram (-) microorganisms, especially Staphylococcus aureus (8). There is very little information in the literature regarding the use of rifamycin in wound care. To the best of our knowledge, there are no studies on the use of rifamycin in antibiotic prophylaxis, this is the first study on this subject. Topical rifamycin use has been reported to be beneficial in hand injuries, controlling infection, and accelerating wound healing (9). In the current study, we evaluated the outcome of using topical rifamycin prophylaxis in obstetric and gynecological operations.

\section{Material and Method}

The study has been approved by the ethics committee of Erzincan University's presidency on 22.08.2017 with the decision numbered 12/02. A total of 100 patients, operated on at our University, were included in this case-control study during the last quarter period of 2017. Operating room reports and file records of the operated patients were screened. A total of 167 patients were identified and those who underwent an abdominal hysterectomy, laparoscopic hysterectomy, and cesarean section were included in the study. Patients were selected in sequential order. Data on age, gender, emergent/elective operations, medical illnesses, body mass index (BMI), type of surgical technique, antibiotic protocol in patients, presence of surgical drain, postoperative seroma, culture results in case of infection within the first ten days and length of follow-up were extracted. Presence of Diabetes mellitus (DM) or immune, or rheumatologic or immunosuppressive disease or received immunosuppressant treatment, receiving antibiotic therapy for local or systemic infection, using anticoagulants or oral contraceptives, patients lost to follow-up were excluded, after exclusion of 67 such cases the study group contained 100 patients. A flow chart of the study design is presented in figure 1 . The surgical site was shaved with an electrical shaver 30 minutes before the operation. The cleaning was done by $10 \%$ povidoneiodine. The 100 study patients were divided into two groups according to their antibiotic use; 50 patients who received only $1 \mathrm{~g}$ cefazolin intraoperatively constituted Group 1, 50 patients who received only $250 \mathrm{mg}$ topical rifamycin over the incision line based on surgeon's preference constituted Group 2. Postoperative antibiotics were not used in both patient groups.
The prophylactic antibiotic regimen we used was $1 \mathrm{~g}$ cefazolin sodium (Cefamezin-IM/IV $\left.{ }^{\circledR}\right) 30$ minutes before surgery. In the rifamycin group, the surgical site was irrigated using rifamycin (Rif ${ }^{\circ} 250 \mathrm{mg} / 3 \mathrm{ml}$ ampoule). The uterine cavity was washed with diluted rifamycin in cesarean section, on the other hand, rifamycin was used to irrigate the Douglas and cuffs in hysterectomy surgeries. Rifamycin was sprayed onto the trocar areas with an injector after the trocars were removed in patients undergoing laparoscopic hysterectomy. In the control group (Group 1) irrigation was performed only with isotonic. The two groups were compared concerning risk for SSI. SSI was evaluated within 10 days following the operation. The presence of infection was verified by obtaining cultures in case of detection of at least one of the signs: fever, warmness, swelling, fluctuation, and redness. In cases of wound infection, the wound was opened and drainage was performed, and the culture was evaluated with a bacteriologic antibiogram. Statistical package program SPSS 20 (IBM Corp. released 2011. IBM SPSS Statistics for Windows, version 20.0, Armonk, NY: IBM Corp.) was used to evaluate the data. Variables mean \pm standard deviation and Median (Maximum-Minimum) percentage and frequency values are used. Also, the homogeneity of the variances from the preconditions of the parametric tests was checked by the "Levene's" test. The assumption of normality was checked by the "Shapiro-Wilk" test. When the differences between the two groups were to be evaluated "Student's t-Test" was used when the parametric test conditions were met. "Mann Whitney-U test" was used when the parametric test conditions were not met. Relationships between categorical variables were analyzed by Fisher's Exact Test and Chi-square test. In cases where the expected frequencies are less than 20\%, the "Monte Carlo Simulation Method" is used to include these frequencies in the analysis. Binary logistic regression analysis was performed. $p<0.05$ was accepted as statistically significant.

\section{Results}

We designed a study that included 100 women. There were no significant differences between groups in terms of age, gravida, parity, and duration of the operation. Demographic analyses are presented in table I. The percentages of abdominal hysterectomy, laparoscopic hysterectomy, elective cesarean section, and emergency cesarean section were 30, 20, 28 , and 22, respectively (Table II).

Figure 1: Flow chart of the study design

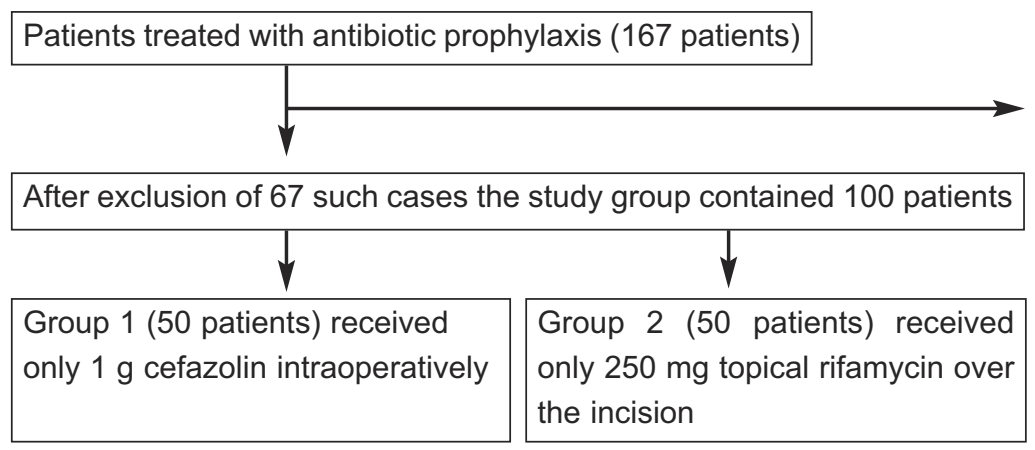

Exclusion criteria (67 patients)
- Presence of Diabetes mellitus
Immune, or rheumatologic or immunosuppressive
disease
- Received immunosuppressant treatment
- Received antibiotic therapy for local or systemic
infection
- Using anticoagulants
- Using oral contraceptives


Table I: Demographic characteristics of patients

\begin{tabular}{llllccc}
\hline GROUP & & $\mathbf{n}$ & Mean & Std. deviation & Std. error mean & $\boldsymbol{p}$ \\
\hline Age & Rifamycin & 50 & 40.86 & 13.75 & 1.94 & 0.546 \\
Gravida & Cefazolin & 50 & 39.14 & 14.60 & 2.06 & 0.27 \\
& Rifamycin & 50 & 4.08 & 1.88 & 0.142 \\
Parity & Cefazolin & 50 & 3.52 & 1.90 & 0.27 & 0.18 \\
& Rifamycin & 50 & 3.06 & 1.28 & 0.22 & 3.18 \\
Operation time/minute & Cefazolin & 50 & 2.80 & 22.51 & 0.547 \\
& Rifamycin & 50 & 66.64 & 22.47 & 3.18 \\
\hline
\end{tabular}

Std. deviation: Standard deviation, Std. error mean: Standard error mean, $p<0.05$ was accepted as statistically significant.

Table II: Clinical and demographic characteristics of patients.

\begin{tabular}{|c|c|c|c|c|c|c|}
\hline & & & \multicolumn{2}{|c|}{ Group } & \multirow{2}{*}{ Total } & \multirow{2}{*}{$p$} \\
\hline & & & Rifamycin & Cefazolin & & \\
\hline \multirow{2}{*}{ Marital status } & Married & $\mathrm{n}$ & 50 & 45 & 95 & \multirow{2}{*}{$0.022^{*}$} \\
\hline & Single & $\mathrm{n}$ & 0 & 5 & 5 & \\
\hline \multirow{2}{*}{ Occupation } & Housewife & $\mathrm{n}$ & 46 & 42 & 88 & \multirow{2}{*}{0.218} \\
\hline & Worker & $\mathrm{n}$ & 4 & 8 & 12 & \\
\hline \multirow{4}{*}{ Operation performed } & Abdominal hysterectomy & $\mathrm{n}$ & 15 & 15 & 30 & \multirow{4}{*}{0.955} \\
\hline & Laparoscopic hysterectomy & $\mathrm{n}$ & 10 & 10 & 20 & \\
\hline & Elective cesarean & $\mathrm{n}$ & 13 & 15 & 28 & \\
\hline & Emergency cesarean & $\mathrm{n}$ & 12 & 10 & 22 & \\
\hline \multirow{2}{*}{ Smoking } & No & $\mathrm{n}$ & 37 & 41 & 78 & \multirow{2}{*}{0.334} \\
\hline & Yes & $\mathrm{n}$ & 13 & 9 & 22 & \\
\hline \multirow{4}{*}{ Education level } & illiterate & $\mathrm{n}$ & 5 & 6 & 11 & \multirow{4}{*}{0.510} \\
\hline & Primary education & $\mathrm{n}$ & 25 & 23 & 48 & \\
\hline & High school & $\mathrm{n}$ & 14 & 10 & 24 & \\
\hline & University & $\mathrm{n}$ & 6 & 11 & 17 & \\
\hline \multirow{2}{*}{ Residence } & Rural & $\mathrm{n}$ & 16 & 34 & 50 & \multirow{2}{*}{$0.001^{* *}$} \\
\hline & Urban & $\mathrm{n}$ & 34 & 16 & 50 & \\
\hline \multirow{2}{*}{ Fewer } & No & $\mathrm{n}$ & 46 & 48 & 94 & \multirow{2}{*}{0.410} \\
\hline & Yes & $\mathrm{n}$ & 4 & 2 & 6 & \\
\hline \multirow{2}{*}{$\begin{array}{l}\text { Surgical site } \\
\text { infections }\end{array}$} & No & $\mathrm{n}$ & 50 & 45 & 95 & \multirow{2}{*}{$0.022^{*}$} \\
\hline & Yes & $\mathrm{n}$ & 0 & 5 & 5 & \\
\hline \multirow{2}{*}{ Pelvic infection } & No & $\mathrm{n}$ & 48 & 50 & 98 & \multirow{2}{*}{0.153} \\
\hline & Yes & $\mathrm{n}$ & 2 & 0 & 2 & \\
\hline \multirow{2}{*}{ Urinary infection } & No & $\mathrm{n}$ & 47 & 48 & 95 & \multirow{2}{*}{0.646} \\
\hline & Yes & $\mathrm{n}$ & 3 & 2 & 5 & \\
\hline \multirow{3}{*}{ Bacterial antibiogram } & S. aureus & $\mathrm{n}$ & 0 & 5 & 5 & \multirow{3}{*}{$0.007^{* *}$} \\
\hline & S. epidermidis & $\mathrm{n}$ & 0 & 2 & 2 & \\
\hline & E. coli & $\mathrm{n}$ & 5 & 0 & 5 & \\
\hline \multirow{4}{*}{ Blood transfusion } & None & $\mathrm{n}$ & 45 & 43 & 88 & \multirow{4}{*}{0.695} \\
\hline & No & $\mathrm{n}$ & 47 & 46 & 93 & \\
\hline & Yes & $\mathrm{n}$ & 3 & 4 & 7 & \\
\hline & Total & $\mathrm{n}$ & 50 & 50 & 100 & \\
\hline
\end{tabular}

${ }^{*} p<0.05,{ }^{* *} p<0.01$. S. aureus: Staphylococcus aureus, S. epidermidis: Staphylococcus epidermidis, E. coli: Escherichia coli. Relationships between categorical variables were analyzed by Fisher's Exact Test and Chi-square test, $p<0.05$ was accepted as statistically significant. 
The prophylactic topical rifamycin reduced the incidence of wound infection compared to cefazoline (Table II). Although there was no statistically significant difference between the groups in terms of fever, pelvic infection, and urinary infection, significant differences were found between the residence, wound infections, and bacteriological antibiogram variables (Table II). SSI was detected in $5(10 \%)$ of the patients who received surgical prophylaxis with cefazolin, whereas SSI was not observed in patients who received antimicrobial prophylaxis with rifamycin $(p=0.022)$. In the logistic regression analysis performed by including BMI, operation time, and surgery type covariates, it was found that topical rifamycin prophylaxis did not reduce the risk of wound infection independently table III.

\section{Discussion}

SSIs after abdominal surgery is a major problem. Many methods have been tried to reduce the incidence of SSIs. The basic principles of antibiotic use against wound infection were firstly introduced by Burke's experimental studies. Burke determined that the surgical area was contaminated during surgery and showed that antibiotic therapy should be initiated before surgery (10). The purpose of doing this is to obtain the concentration of antibacterial drug at a dose that will leave the bacteria in the wound tissue ineffective while the wound is contaminated with the bacteria (10). Local application of topical antibiotics is an attractive method because it has the potential to cause fewer adverse systemic side effects due to less systemic exposure by giving high-dose antibiotics to the surgical site. However, there is no high-quality clinical evidence supporting this practice in surgical literature (11). Topical rifamycin can be used in some clinics in Turkey for antibiotic prophylaxis but there is no trial in the international literature related to this application. To our knowledge, our study is the first to clinically demonstrate that using topical rifamycin as a prophylactic antibiotic in gynecological and obstetric surgery. Clinical guidelines on the use of prophylactic antibiotics for gynecological surgery by the American College of Obstetricians and Gynecologists (ACOG) were published in 2009 (12). While the guidelines are extremely useful for gynecological surgeons, most of the guidelines have different study designs and include work in other fields. The guidelines are based on consensus and expert opinion. For this reason, we believe that the evidence levels of available evidence regarding the use of prophylactic antibiotics in gynecological procedures are poor. The application of prophylactic antibiotics in gynecological and obstetric operations shows some differences compared to other surgical branches. Firstly, almost all of the obstetric cases and most of the gynecological cases are young, healthy people. Secondly, although the lower genital area is contaminating with a large number of aerobic and anaerobic microorganisms, there are rarely any antibiotic-resistant gram-negative organisms, except in some special cases. Thirdly infections may develop mildly or moderately after operations performed within or near this contaminant site, but complications such as bacteremia, abscess formation, and death are rarely encountered. For all these reasons, it seems reasonable to perform topical antibiotic prophylaxis instead of systemic antibiotic prophylaxis in gynecological and obstetric surgery. Currently, surgical wounds are classified as clean, clean-contaminated, contaminated, and dirty-infected wounds (13). As it's known, cesarean and hysterectomy are in the class of clean contaminants so antibiotic prophylaxis is recommended for these surgeries. All patients in our study were in a clean-contaminated class. Agents responsible for surgical wound infections are usually endogenous floradors. The most common microorganisms associated with superficial wound infections are Staphylococcus aureus and Staphylococcus epidermidis. Deep infections often involve a variety of gram-negative organisms, such as Escherichia coli and Klebsiella spp. If clinics detect specific pathogens of their own, prophylaxis should be provided for these organisms. The commonly used antibiotic is first-generation cephalosporins. There is limited information about the role of topical antibiotics in preventing SSIs. Rifamycin is a semisynthetic antibiotic with strong bactericidal effects on bacteria including staphylococcus aureus but data on its use in surgical prophylaxis are limited (8). Saydam and colleagues reported in their experimental studies that rifamycin is inexpensive and effective on Staphylococcus aureus and Staphylococcus epidermidis for full-thickness wound care (14), but there is not yet a clinical study supporting this experimental study. Our work is

Table III: Multivariate analysis of variables

\begin{tabular}{llll}
\hline Variable & Odds Ratio & 95\% Confidence Interval & $\boldsymbol{p}$ \\
\hline Rifamycin vs Cefazolin (Ref.: Cefazolin) & 0 & NC & 0.997 \\
Age $\leq 40$ vs Age $\geq 41$ (Ref.: Age $\geq 41$ ) & 0.706 & $0.007-72.358$ \\
Gravida $\leq 3$ vs Gravida $\geq 4$ (Ref.: Gravida $\geq 4)$ & 0.86 & $0.078-9.512$ & 0.883 \\
Parity $\leq 2$ vs Parity $\geq 3$ (Ref.: Parity $\geq 3$ ) & 2.036 & $0.105-39.5$ & 0.602 \\
Operation Type (Elective vs Emergency) (Ref.:Emergency) & 0.207 & $0.011-3.867$ \\
Not smoking vs. Smoking (Ref.:Smoking) & 0.934 & $0.061-14.217$ & 0.292 \\
Operation Time $\leq 65$ min. vs. $\geq 66$ min. (Ref.: $\geq 66$ min.) & 0.749 & $0.009-61.644$ & 0.961
\end{tabular}

Multivariate analyzes method: Binary logistic regression, Dependent: Surgical site infection, Ref: Reference, NC: Can not be calculated by SPSS, vs: versus, Min: Minutes 
the first clinical trial to support this study. Iselin et al. (9) reported that rifamycin was superior to povidone-iodine in terms of infection prophylaxis of extremity injuries. Weber et al reported a reduction in the risk of catheter-associated infection in children with minocycline/rifamycin coated catheter use (15). In a recent study conducted by Karuserci et al., rifamycin, povidone-iodine, and saline were compared in preventing superficial incisional infections, the results were found to be significant in favor of the rifamycin and povidone-iodine group (16). Similarly, Neri et al. demonstrated that topical application of rifamycin to the umbilicus during pre-, intra-, and postoperative periods decreased infective complications after laparoscopic cholecystectomy (17). In our work, we have supported these studies. Our results showed that SSIs occur less frequently in rifamycin-treated patients than in the control group. In a study conducted by Neri et al, they reported that topical rifamycin use in laparoscopic cholecystectomy operations reduced umbilical port wound infections (17). On the other hand; Aygun et al. Reported that topical rifamycin used after cardiovascular surgery had no protective effect for sternal wound infections (18). The reduction of SSIs with the use of topical rifamycin is the main result of our study. The use of topical rifamycin is effective but does not imply that systemic antibiotics should replace prophylaxis. The use of rifamycin would aid in systemic antibiotic prophylaxis.

This study has several limitations. First of all, this is a case-control study with small sample size. Secondly, although the study was a case-control study, post hoc power analysis could be done, but it was not, this is another of the limitations of the study.

\section{Conclusion}

As a result, SSIs were significantly reduced when the incision line was irrigated with rifamycin. However, multi-center studies with more patients are needed to investigate the subject.

\section{Declarations}

Acknowledgment: We are grateful to all participants and their families who spent their precious time and participated in this research program. We are also thankful for the tireless efforts of the research team members.

Competing interests: The authors declare that they have no competing interests.

Funding: No person/organization financially supports the study.

All participants signed informed written consent before being enrolled in the study.

All procedures were performed according to the Declaration of Helsinki.

Availability of data and materials: The datasets and code used and/or analyzed during the current study are available from the corresponding author on reasonable request.
Authors' contributions: $N A, M K$, and NGK: raised the presented idea. NA, MK, and NGK: designed the study. MK: conducted the analyses. NGK. and MK: developed the first draft of the manuscript. All authors contributed to the writing of the paper, and have read and approved the final manuscript. CT, $A B, C C$ : conducted the population study, analyzed and interpreted the data, and drafted the manuscript. MK: participated in data analysis, interpretation, and draft revision. $A B$, and $C C$ : participated in data collection and result interpretation. $C T, A B$, and CC: assisted with data collection and analysis. $C C, M K$, and NA: designed the study and critically revised the manuscript. All authors read and approved the final manuscript.

\section{References}

1. Mangram AJ, Horan TC, Pearson ML, Silver LC, Jarvis WR. Guideline for prevention of surgical site infection, 1999. Centers for Disease Control and Prevention (CDC) Hospital Infection Control Practices Advisory Committee. Am J Infect Control. 1999;27(2):97-132; quiz 133-4; discussion 96. PMID: 10196487.

2. Horan TC, Gaynes RP, Martone WJ, Jarvis WR, Emori TG. CDC definitions of nosocomial surgical site infections, 1992: a modification of CDC definitions of surgical wound infections. Am J Infect Control. 1992;20(5):271-4. Doi: 10.1016/s0196-6553(05)80201-9.

3. Garner JS, Jarvis WR, Emori TG, Horan TC, Hughes JM. CDC definitions for nosocomial infections, 1988. Am J Infect Control. 1988;16(3):128-40. Doi: 10.1016/01966553(88)90053-3.

4. Anderson DJ, Podgorny K, Berríos-Torres SI, Bratzler DW, Dellinger EP, Greene L, et al. Strategies to prevent surgical site infections in acute care hospitals: 2014 update. Infect Control Hosp Epidemiol. 2014;35(6):605-27. Doi: $10.1086 / 676022$.

5. Nichols RL. Preventing surgical site infections: a surgeon's perspective. Emerg Infect Dis. 2001;7(2):220-4. Doi: $10.3201 /$ eid0702.010214.

6. Zumla A Mandell, Douglas, and Bennett's principles and practice of infectious diseases. Lancet Infect Dis. 2010;10(5):303-4. Doi: 10.1016/S1473-3099(10)70089-X

7. Kremer KM, Foster RT, Drobnis EZ, Hyde KJ, Brennaman LM. Non-indicated use of prophylactic antibiotics in gynaecological surgery at an academic tertiary medical centre. J Obstet Gynaecol. 2018;38(4):543-547. Doi: 10.1080/01443615.2017.1371119.

8. Park DH, Lee SA, Jeong HJ, Yoo TH, Kang SW, Oh HJ. Rifampicin-induced minimal change disease is improved after cessation of rifampicin without steroid therapy. Yonsei Med J. 2015;56(2):582-5. Doi: 10.3349/ymj.2015. 56.2.582.

9. Iselin F, Audren JL, Gouet O, Hautefort E, Peze W, Pradet G. Comparative study of the effects of a local antibiotic 
and a local antiseptic in emergency hand surgery. Ann Chir Main Memb Super. 1990;9(1):65-71. French. Doi: 10.1016/s0753-9053(05)80441-7.

10. Burke JF. The effective period of preventive antibiotic action in experimental incisions and dermal lesions. Surgery. 1961;50:161-8. PMID: 16722001.

11. Fleischman AN, Austin MS. Local intra-wound administration of powdered antibiotics in orthopaedic surgery. J Bone Jt Infect. 2017;2(1):23-28. Doi: 10.7150/jbji.16649.

12. ACOG practice bulletin No. 104: antibiotic prophylaxis for gynecologic procedures. Obstet Gynecol. 2009; 113(5):1180-1189. Doi: 10.1097/AOG.0b013e3181 a6d011.

13. Yerushalmy A, Reches A, Lessing JB, Schechner V, Carmeli Y, Grisaru D. Characteristics of microorganisms cultured from infected wounds post-hysterectomy. Eur J Obstet Gynecol Reprod Biol. 2008;141(2):169-72. Doi: 10.1016/j.ejogrb.2008.07.024.

14. Saydam I, Yilmaz S, Seven E. The effect of nitrofurasone and rifampycine on full thickness wound healing. CÜ Tip Fak Derg. 2005;27:113-20.
15. Weber JM, Sheridan RL, Fagan S, Ryan CM, Pasternack MS. Tompkins RG. Incidence of catheter-associated bloodstream infection after introduction of minocycline and rifampin antimicrobial-coated catheters in a pediatric burn population. J Burn Care Res. 2012;33(4):539-43. Doi: 10.1097/BCR.0b013e31823c4cd5.

16. Karuserci OK, Sucu S, Ozcan HC, Tepe NB, Ugur MG, Guneyligil $\mathrm{T}$, et al. Topical rifampicin versus povidoneiodine for the prevention of incisional surgical site infections following benign gynecologic surgery: a prospective, randomized, controlled trial. New Microbiol. 2019;42(4):205-209. PMID: 31524943.

17. Neri V, Fersini A, Ambrosi A, Tartaglia N, Valentino TP. Umbilical port-site complications in laparoscopic cholecystectomy: role of topical antibiotic therapy. JSLS. 2008;12(2):126-32. PMID: 18435883

18. Aygun F, Kuzgun A, Ulucan S, Keser A, Akpek M, Kaya M. The protective effect of topical rifamycin treatment against sternal wound infection in diabetic patients undergoing on-pump coronary artery bypass graft surgery. Cardiovasc J Afr. 2014;25(3):96-9. Doi: 10.5830/CVJA2014-008. 